\title{
A basin-free spherical shape as outcome of a giant impact on asteroid
}

\section{Hygiea}

P. Vernazza ${ }^{1}$, L. Jorda ${ }^{1}$, P. Ševeček ${ }^{2}$, M. Brož ${ }^{2}$, M. Viikinkoski ${ }^{3}$, J. Hanuš ${ }^{2}$, B. Carry $^{4}$, A.

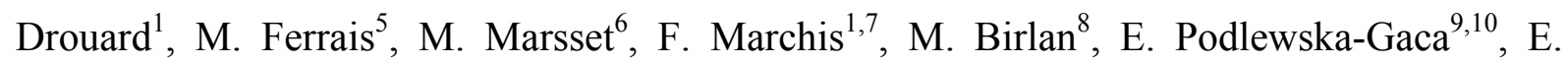
Jehin $^{5}$, P. Bartczak ${ }^{9}$, G. Dudzinski ${ }^{9}$, J. Berthier ${ }^{8}$, J. Castillo-Rogez ${ }^{11}$, F. Cipriani ${ }^{12}$, F. Colas ${ }^{8}$,

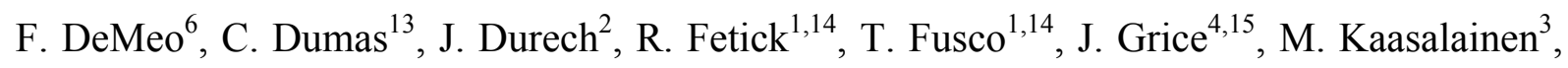
A. Kryszczynska ${ }^{9}$, P. Lamy ${ }^{1}$, H. Le Coroller ${ }^{1}$, A. Marciniak ${ }^{9}$, T. Michalowski ${ }^{9}$, P. Michel $^{4}$, N.

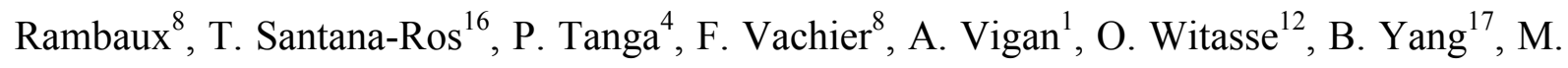
Gillon $^{5}$, Z. Benkhaldoun ${ }^{18}$, R. Szakats $^{19}$, R. Hirsch ${ }^{9}$, R. Duffard ${ }^{20}$, A. Chapman ${ }^{21}$, J. L. Maestre $^{22}$

${ }^{1}$ Aix Marseille Université, CNRS, LAM (Laboratoire d'Astrophysique de Marseille) UMR 7326, 13388, Marseille, France

${ }^{2}$ Institute of Astronomy, Charles University, Prague, V Holešovičká ch 2, 18000, Prague 8, Czech Republic

${ }^{3}$ Mathematics and statistics, Tampere University, PO Box 553, 33101, Tampere, Finland

${ }^{4}$ Université Côte d'Azur, Observatoire de la Côte d'Azur, CNRS, Laboratoire Lagrange, 06304, Nice Cedex 4, France

${ }^{5}$ Space sciences, Technologies and Astrophysics Research Institute, Université de Liège, Allée du 6 Août 17, 4000, Liège, Belgium

${ }^{6}$ Department of Earth, Atmospheric and Planetary Sciences, MIT, 77 Massachusetts Avenue, Cambridge, MA 02139, USA

${ }^{7}$ SETI Institute, Carl Sagan Center, 189 Bernado Avenue, Mountain View CA 94043, USA 
${ }^{8}$ IMCCE, Observatoire de Paris, 77 avenue Denfert-Rochereau, 75014 Paris Cedex, France

${ }^{9}$ Astronomical Observatory Institute, Faculty of Physics, Adam Mickiewicz University, Słoneczna 36, 60-286 Poznań, Poland

${ }^{10}$ Institute of Physics, University of Szczecin, Wielkopolska 15, 70-453 Szczecin, Poland

${ }^{11}$ Jet Propulsion Laboratory, California Institute of Technology, 4800 Oak Grove Drive, Pasadena, CA 91109, USA

${ }^{12}$ European Space Agency, ESTEC - Scientific Support Office, Keplerlaan 1, Noordwijk, 2200 AG, The Netherlands

${ }^{13}$ TMT Observatory, 100 W. Walnut Street, Suite 300, Pasadena, CA 91124, USA

${ }^{14}$ ONERA, The French Aerospace Lab BP72, 29 avenue de la Division Leclerc, 92322 Chatillon Cedex, France

${ }^{15}$ Open University, School of Physical Sciences, The Open University, MK7 6AA, UK

${ }^{16}$ Departamento de Física, Ingeniería de Sistemas y Teoría de la Señal, Universidad de Alicante, E-03080 Alicante, Spain

${ }^{17}$ European Southern Observatory (ESO), Alonso de Cordova 3107, 1900 Casilla Vitacura, Santiago, Chile

${ }^{18}$ Oukaimeden Observatory, High Energy Physics and Astrophysics Laboratory, Cadi Ayyad University, Marrakech, Morocco

${ }^{19}$ Konkoly Observatory, Research Centre for Astronomy and Earth Sciences, Hungarian Academy of Sciences, Konkoly Thege 15-17, H-1121 Budapest, Hungary

${ }^{20}$ Instituto de Astrofísica de Andalucía (CSIC), Glorieta de la Astronomía S/N, 18008Granada, Spain

${ }^{21}$ Buenos Aires, Argentina

${ }^{22}$ Observatorio de Albox (IAU code: Z90), Apdo. correos, 63. 04800 Albox - Almeria, Spain 
Hygiea is the fourth largest main belt asteroid and the only known asteroid whose surface composition appears similar to that of the dwarf planet $\operatorname{Ceres}^{1,2}$, suggesting a similar origin for these two objects. Hygiea suffered a giant impact more than 2 Gyr ago $^{3}$ that is at the origin of one of the largest asteroid families. However, Hygeia has never been observed with sufficiently high resolution to resolve the details of its surface nor to constrain its size and shape. Here, we report high angular resolution imaging observations of Hygiea with the VLT/SPHERE instrument ( 20 mas at $600 \mathrm{~nm})$ that reveal a basin-free nearly spherical shape with a volume equivalent radius of $217( \pm 7)$ $\mathrm{km}$, implying a density of $1944( \pm 250 ; 1$-sigma $) \mathrm{kg} / \mathrm{m}^{3}$. In addition, we determined a new rotation period for Hygiea, $P \sim 13.8 h$, that is half of the currently accepted value. Numerical simulations of the family forming event show that Hygiea's spherical shape and family can be explained by a collision with a large projectile (Diameter $75-150 \mathrm{~km}$ ). By comparing Hygiea's sphericity with that of other solar system objects, it appears that Hygiea is nearly as spherical as Ceres, opening a possibility for this object to be reclassified as a dwarf planet.

Albeit being an easy target for ground based observations owing to its large angular diameter, Hygiea is the least studied of the four asteroids with diameters greater than $400 \mathrm{~km}$ (Ceres, Pallas, Vesta and Hygiea; Fig. 1), whose large sizes may have allowed them to reach hydrostatic equilibrium early in their history. It follows that a number of its basic physical properties, such as its shape and spin state have not yet been reliably constrained.

To constrain these physical properties, we performed - as part of our ESO large program $^{4}$ - high angular resolution imaging observations of Hygiea with the SPHERE instrument on the Very Large Telescope (Paranal Observatory, Chile) at 12 different epochs in 2017 and 2018. We used the new-generation visible adaptive optics ZIMPOL $^{5}$ in narrow 
band imaging mode $\left(\mathrm{N} \_\mathrm{R}\right.$ filter; central wavelength $\left.=645.9 \mathrm{~nm}\right)$. In order to restore the optimal angular resolution of each reduced image, we used the MISTRAL myopic deconvolution algorithm ${ }^{6}$ alongwith a parametric Point Spread Function ${ }^{7}$. We then applied the All-Data Asteroid Modeling $\left(\mathrm{ADAM}^{8}\right)$ algorithm to our set of deconvolved images to reconstruct the $3 \mathrm{D}$ shape model and the spin of Hygiea. The shape reconstruction was complicated by discernible albedo variegation apparent in the images (see Methods). To take into account such phenomenon, the relative brightness of each facet with respect to the surrounding ones was treated as a free parameter (we allowed a maximum variegation of $\pm 30 \%)$ and we further defined a smoothing operator as a regularization term to prevent large deviations between neighboring facets. The comparison between the twelve adaptive optics epochs and the corresponding shape model projections is shown in Fig. 2.

Our best fits yielded semi-axes of $225 \pm 5 \mathrm{~km}, 215 \pm 5 \mathrm{~km}$, and $212 \pm 10 \mathrm{~km}$ and a volume equivalent radius of $217 \pm 7 \mathrm{~km}$. We found a rotational pole of right ascension $319 \pm$ $3^{\circ}$, declination $-46 \pm 3^{\circ}$ and a rotation period of $13.82559 \pm 0.00005 \mathrm{~h}$, that is half of the previously reported and widely accepted value ${ }^{9}$. Our rotation period is compatible both with all lightcurves acquired so far for Hygiea including the ones acquired with the TRAPPIST telescopes in parallel to our SPHERE observations (supplementary figure 1) and the SPHERE images. The axial ratios including their uncertainties appear compatible with the equilibrium MacLaurin spheroid. The specific angular momentum $L_{-}$norm $=\mathrm{L} / \operatorname{sqrt}\left(\mathrm{G} \mathrm{M}^{3} \mathrm{R}\right)=0.070 \pm$ 0.002 is lower than the bifurcation point $(0.304)$ where the equilibrium figure becomes a triaxial Jacobi ellipsoid ${ }^{10}$.

Our shape and our best estimate of Hygiea's mass, $(8.32 \pm 0.80) \times 10^{19} \mathrm{~kg}$ (supplementary figure 2 and supplementary table 3), yield a density of $1944 \pm 250 \mathrm{~kg} / \mathrm{m}^{3}$. Such density is compatible, within errors, with Ceres' density ${ }^{11}\left(2161.6 \pm 2.5 \mathrm{~kg} / \mathrm{m}^{3}\right)$. Note that the reaccumulation process following the giant impact at the origin of the family (see 
hereafter) may have trigggered some level of macroporosity and the original density of Hygiea may be even closer to that of Ceres. The high water fraction inferred in both cases alongwith their similar spectral properties ${ }^{1,2}$ imply a formation location beyond the snowline for these two bodies.

We observed Hygiea with sub-Earth latitudes near $50^{\circ} \mathrm{S}$ (first epoch) and $24^{\circ} \mathrm{S}$ (second epoch) so that the visible surface extended from $66^{\circ} \mathrm{N}$ through $90^{\circ} \mathrm{S}$, leading to $\sim 95 \%$ surface coverage. Surprisingly, none of our images and their associated contours (supplementary figure 3) revealed the large impact basin expected from the large size of the Hygiea family ${ }^{3,12}$ (volume-equivalent diameter $\left(D_{\text {eq }}\right)$ of the family members $\sim 100 \mathrm{~km}$; see Methods). In comparison, Vesta possesses a large impact basin that is clearly observable from the ground $^{13,7}$ (Fig. 1) although its family is smaller in volume than Hygiea's family by a factor of $\sim 8\left(\mathrm{D}_{\text {eq }} \sim 50 \mathrm{~km}\right)^{12}$. To quantify the overall absence of a large basin on Hygiea, we fit Hygiea's 3D shape model with an ellipsoid and subsequently measured the radial difference between the two shapes. We also calculated the volume fraction of excavated material as |Volume_Body - Volume_Ellipsoid|/ Volume_Body. We performed the same calculations for Ceres and Vesta. Our calculations show that the large-scale topography of Hygiea is similar to that of Ceres, implying a global lack of large impact basin across its surface. They also reveal that - similarly to Ceres - Hygiea's shape is very close to that of an ellipsoid. In the case of Vesta, the existence of a large depression is clearly observed in the histogram (supplementary figure 4).

To investigate the origin of Hygiea's nearly spherical shape as well as the absence of a large impact basin, we used a smoothed particle hydrodynamics (SPH) code ${ }^{14-16}$ to simulate the family-forming event. Our code is well adapted to simulate collisions of rotating and selfgravitating asteroids. We assumed monolithic basaltic material, the Tillotson equation of state $^{17}$, the von Mises yield criterion ${ }^{18}$ to account for plastic deformations and the Grady-Kipp 
model $^{19}$ for fragmentation. The self-gravity has been implemented using the Barnes-Hut $\operatorname{algorithm}^{20}$. All input parameters are listed in supplementary table 5. Prior to running the simulations, our code was tested against previous studies ${ }^{14}$, and we also carefully verified the stability of rotating objects as well as the validity of the gravity approximation by comparing it to the 'brute-force' approach.

We performed a large number of simulations testing various projectile diameters $\left(d_{i m p}\right.$ range: $70-150 \mathrm{~km}$ ), impact angles ( $\phi_{\mathrm{imp}}$ range $: 15-60 \mathrm{deg}$ ), and initial rotation periods for the target $\left(\mathrm{P}_{\mathrm{pb}}\right.$ range: $\left.3-\infty \mathrm{h}\right)$. Large values for the projectile diameter were required to match the large size of the Hygiea family. We further used a range of impact speeds from 5 up to 7 $\mathrm{km} / \mathrm{s}$. Both fragmentation and reaccumulation phases were computed by the SPH algorithm to resolve the shape of the largest remnant (i.e. Hygiea). Only for the final reaccumulation we switched to a more efficient N-body algorithm, using hard-sphere and perfect-merging approximations, to obtain a synthetic family and its size frequency distribution (SFD). The numerical model is described in detail in Methods.

A first outcome of our simulations is that Hygiea's final shape is highly spherical, regardless the diameter of the impactor (in the $75-150 \mathrm{~km}$ size range) and the impact angle (Fig. 3). In particular, all pre-existing surface features have been erased implying that the observed absence of a large impact basin on Hygiea is a natural outcome of the family forming impact. We further used the SFD of the observed family to better constrain the parameters of the giant collision. It appears that the observed SFD can be matched either by head-on (0-30 deg) $d_{\text {imp }}=75 \mathrm{~km}$ impacts, or alternatively oblique $(30-60 \mathrm{deg}) \mathrm{d}_{\text {imp }}=150 \mathrm{~km}$ impacts, although only the head-on impacts form one or few intermediate-sized (40 $\mathrm{km}<\mathrm{D}<100 \mathrm{~km}$ ) fragments; no such fragments are formed for impact angles greater than $45^{\circ}$. Given that the second largest body of the family [(1599) Giomus; see Methods] is indeed an intermediate-sized fragment, the head-on impact is more plausible. It follows that the 
impactor had likely $\mathrm{d}_{\mathrm{imp}} \sim 100 \mathrm{~km}$. Our simulations imply that the impact fully damaged the parent body and resulted in substantial reaccumulation ${ }^{21}$. When Hygiea formed, macroscopic oscillations drove the material to behave as a fluid ${ }^{22}$, naturally resulting in the formation of a rotational equilibrium nearly spherical object (Fig. 3). Accordingly, the effective friction of the damaged material had to be negligible for Hygiea (see Methods). Some departures from a rotational equilibrium can occur only if the material regains its strength, e.g. when acoustic fluidization is stopped ${ }^{23,24}$. Indeed, we detect global oscillations of the shape in our simulations (see supplementary figure 5), which logically occur on the keplerian time scale, i.e. 2.4 hours. Using a,b,c for semi-axes of a dynamically equivalent ellipsoid, we can explain the observed $b / a$ and $c / b$ ratios provided the fluidization stopped after approximately 4 hours. In contrast to Hygiea, the Rheasilvia basin on Vesta resulted from an impact by a $\mathrm{D} \sim 65 \mathrm{~km}$ sized projectile ${ }^{25}$. In this case, we suppose that, as Vesta is $\sim 3$ times more massive than Hygeia, the impact energy was not sufficient to completely shatter it and the collision ended up being an excavation event.

The nearly spherical shape of Hygiea led us to evaluate the possibility to classify this object as a dwarf planet. Any main belt asteroid satisfies right away three of the four characteristics required for an object being labelled a dwarf planet, namely a celestial body that (a) is in orbit around the Sun, (b) has not cleared the neighbourhood around its orbit, and (c) is not a satellite. The last requirement is to have sufficient mass for its self-gravity to overcome rigid body forces so that it assumes a hydrostatic equilibrium nearly round shape. To properly quantify this last and essentially main criterion, we measured the sphericity ${ }^{26}$ of Hygiea (see Methods) for comparison with that of the terrestrial planets, the two dwarf planets Pluto and Ceres, and a few asteroids (Fig. 4). It appears that Hygiea is nearly as spherical as Ceres $\left(\psi_{\text {Hygiea }} \sim 0.9975 ; \psi_{\text {Ceres }} \sim 0.9988\right)$. Hygiea could thus be classified as a dwarf planet, so far 
the smallest in the solar system. We anticipate the discovery of several new dwarf planet candidates when 3D shape models become available for $\mathrm{D}>400 \mathrm{~km}$ trans-Neptunian objects.

\section{Methods}

\section{Revision of Hygiea's rotation period}

As part of our ESO large program ${ }^{4}$ (ID 199.C-0074; PI: P. Vernazza), we acquire complementary lightcurves when the pole solution of our target is not well constrained and/or when we are not able to reconstruct its $3 \mathrm{D}$ shape with $\mathrm{ADAM}^{8}$ possibly indicating a wrong estimate of its pole solution or of its rotation period. This is exactly the case for Hygiea. Since 1991 (ref 9), multiple authors have all reported a rotation period of $27.6 \mathrm{~h}$ for Hygiea ${ }^{27}$, but there has always been a lack of densely sampled phased lightcurves for this object.

We therefore planned our observations assuming a $27.6 \mathrm{~h}$ rotation period and we observed Hygiea with TRAPPIST-North and - South $^{28}$ over a $\sim 40$ nights timeframe. The phased lightcurve started to show an ordinary double-sinusoidal shape as our observations were going on. However, the lightcurve appeared to be perfectly symmetrical which is very unlikely. We then phased the data using the half period of $\sim 13.8 \mathrm{~h}$, which produced a very convincing fit with a single peak lightcurve (supplementary figure 1). Assuming this new rotation period, we were able to reconstruct Hygiea's 3D shape model as well as to constrain its spin. In addition, the phasing of our VLT/SPHERE images acquired at several epochs became correct with such new rotation period which wasn't the case with the older one.

\section{How round is Hygiea?}




\section{Contour extraction}

We used a first approach, namely contour extraction ${ }^{7}$, in order to highlight the sphericity of Hygiea. We compare in supplementary figure 3 the contours of our Hygiea images with those of a sphere, revealing - on average - a minimal difference between the two. It is important to stress that the contours obtained with VLT/SPHERE are precise at the pixel level ${ }^{7}$

\section{Calculation of the sphericity}

To constrain Hygiea's sphericity and compare it to that of other solar system bodies including planets and minor bodies (asteroids, comets), we applied a sphericity formula ${ }^{26}$ to our $3 \mathrm{D}$ shape model. Following this formula, the sphericity is a function of the surface area and of the volume. However, the surface area is very sensitive to the surface topography and of the resolution of the 3D shape model. Therefore, performing a direct comparison of the sphericity of various objects having very different 3D shape model resolutions and/or topographies would lead to incorrect results. To overcome this problem and in order to perform a self consistent comparison, we computed the real spherical harmonic expansion coefficients $\left(10^{\text {th }}\right.$ order) of the 3D shape model for each object ${ }^{4,29-40}$ (Pettengill et al. 1991, Thomas et al. 1994, Hudson et al. 2000, Ostro et al. 2000, Smith et al. 2001, Jorda et al. 2012, Preusker et al. 2012, Jaumann et al. 2012, Farnham 2013, Preusker et al. 2014, 2016, Vernazza et al. 2018, Viikinkoski et al. 2018). By doing so, we produced 3D shape models that reproduce well the overal shape of our objects ignoring the small scale topographic variations. An example of the procedure is highlighted in supplementary figure 6. As a final step, we applied the formula of the sphericity to these spherical harmonics models. 


\section{Hygiea's reflectance map}

The best-quality SPHERE images were combined together into a cylindrical-projection map in order to study the main geological features of Hygiea. We call it a reflectance map because it contains both albedo and shadow information. Indeed, the limited number of observed geometries and the resolution of the images do not allow to accurately correct for illumination of local topography. As a consequence, we cannot always separate albedo information from shadowing effects.

The quality of each sequence of observations was evaluated according to three criteria: 1) the angular size of Hygiea at the time of the observation, 2) the presence, or not, of deconvolution artefacts in the images, and 3) the consistency of the location of the main albedo features on the surface of Hygiea across the full sequence of images. According to these criteria, the first two epochs of observations, 2017-06-23 and 2017-07-20 were found to provide the highest image quality. The images for these two epochs also exhibit the highest variability in reflectance seen across the surface of Hygiea, and include most of its main albedo features. We therefore chose to use only these images to maximize the resolution and reliability of our map, despite the fact that they only sample about one third of the total surface covered by our complete set of observations.

A photometric correction was applied to each image in order to correct the overall illumination gradient ${ }^{7}$. The asteroidocentric longitude and latitude of each pixel was measured using the ADAM shape model, and its value projected using an equidistant cylindrical projection. The individual maps built from the complete set of selected images were then combined together, using their overlapping regions to adjust their brightness level ${ }^{7}$. The combined map was finally normalized to the average geometric albedo of Hygiea of $7.2 \%$. 
The resulting reflectance map is shown in supplementary figure 7 . It exhibits a wide range of values, with more than $20 \%$ variability with respect to the average, though shadowed regions enhance this variability. Several bright spots are clearly identifiable, the brightest one, located near $\lambda=290^{\circ}, \phi=-30^{\circ}$, showing a $10 \%$ brightness enhancement with respect to the average reflectance. The large dark region at $\lambda=60^{\circ}, \phi=0^{\circ}$ is most likely a shadowed region, as it is located near the asteroid limb on the second sequence of images.

For comparison, we further show a reflectance map of Ceres (supplementary figure 7), built from our SPHERE observations following the same method as described above for Hygiea. Ceres was observed at one epoch as benchmark target for our observing program, the NASA Dawn mission providing us with the ground truth for that object. Similarly to Hygiea, we used only the best-quality image acquired for that object when building its map. This image contains Ceres' main albedo feature, the bright spot located in the Occator crater. Ceres is slightly brighter than Hygiea in average albedo ( $\mathrm{pv}=0.09$ versus $\mathrm{pv}=0.07$ ). The range of reflectance values revealed by our observations for these two bodies is very similar, with about $20 \%$ variability. Ceres' bright spot in the Occator crater, located around $\lambda=240^{\circ}, \phi=$ $20^{\circ}$, shows a $20 \%$ brightness enhancement with respect to Ceres' average. To conclude, alike for the density and the spectral properties, the reflectance/albedo properties of Hygiea and Ceres are highly similar.

\section{Cratering on Hygiea}

From our set of images, we could identify only two unambiguous craters, with respective diameters of $180 \pm 15 \mathrm{~km}$ and $97 \pm 10 \mathrm{~km}$ (supplementary figure 8). This low number of identified craters contrasts with the large number of craters recognized at the surface of Pallas 
(Fig. 1) and that of (4) $\operatorname{Vesta}^{7}$ and (7) $\mathrm{Iris}^{41}$. Whereas this may be understood as Hygiea'surface being younger than that of the aforementioned bodies, it is unlikely to be the only explanation given that Hygiea's surface age (i.e. estimated formation time of the family) is estimated to be at least 3 Gyrs old ${ }^{3}$. Both the crater morphology and to a lesser extent the reflectance properties of the surface play an important role in the contrast between the crater rim and crater floor. Whereas bowl shaped craters will be easily identifyable from the ground leading to a clear contrast between the crater floor/walls and the crater rim, the same won't be true in the case of complex craters with a flat floor. Most likely, our observations imply a paucity of large ( $\mathrm{D}>30 \mathrm{~km}$ which corresponds to our detection limit) bowl shaped craters in the case of Hygiea. This is an additional common feature between Hygiea and Ceres. In the case of Ceres, the Dawn mission has unambiguously revealed a heavily cratered surface ${ }^{42}$ where most $\mathrm{D}>10-15 \mathrm{~km}$ craters are'nt bowl shaped but flat floored. By analogy with Ceres, this strongly supports the presence of water ice in the subsurface of Hygiea. The presence of water ice in the subsurface would also favor the relaxation of the surface topography as observed on Ceres ${ }^{43}$ thus rending the remote sensing identification of craters on Hygiea more difficult.

\section{Identifying the members of the Hygiea family}

Prior to running the SPH simulations, we carefully identified the Hygiea family members using the proper elements ${ }^{44}$ and the hierarchical clustering method ${ }^{45}$, with the limit relative velocity $\mathrm{v}_{\text {cut }}=60 \mathrm{~m} / \mathrm{s}$. We further used physical data to remove interlopers with incompatible spectra (supplementary figure 9 and supplementary table 4), color (using SDSS data ${ }^{46}$ ) or albedo (using WISE $^{47}$ and $\mathrm{AKARI}^{48}$ data). We found 6857 family members and constructed their size-frequency distribution (SFD). Besides the usual largest remnant (Hygiea), there is 
one intermediate-sized asteroid, namely (1599) Giomus with $\mathrm{D}=46 \mathrm{~km}$ whose near-IR spectrum is compatible with the one of Hygiea (supplementary figure 9). By summing up masses of fragments, we estimate the mass ejected during the collision is at least $1.7 \%$ of the mass of (10) Hygiea. In comparison, the ejected mass of the Vesta family makes up only 0.5 $\%$ of (4) Vesta, suggesting the Hygiea-forming impact was substantially more energetic.

\section{Numerical model}

Impact simulations have been carried out using our SPH/N-body code OpenSPH. The code can perform both SPH and N-body simulations. It thus allows to run a whole simulation, from an initial fragmentation to a final reaccumulation. In all simulations presented here, the duration of the SPH simulation is $t_{\mathrm{SPH}}=24$ hours, which is sufficient for the largest remnant (as well as for the largest fragments) to gain a well-defined shape and damp any macroscopic oscillations. We then follow up with the N-body simulation for another $t_{\mathrm{N} \text {-body }}=10$ days in order to obtain the final SFD of the synthetic family. The hand-off between the SPH and Nbody parts is done by simply changing the solver and modifying the particle radii, $R_{i}=$ $\left[3 M_{i} /(4 \pi \rho)\right]^{1 / 3}$, in order to convert smoothed particles into hard spheres while preserving their masses and volumes.

The SPH solver computes particle accelerations due to the stress tensor and self-gravity, shock heating, material yielding and fragmentation. It further includes the artificial viscosity term for proper treatment of shocks, the artificial stress to suppress tensile instabilities and the correction tensor for consistent bulk rotation ${ }^{49}$. The code can use either a frictionless rheology (von Mises criterion) or a more complex Drucker-Prager rheology ${ }^{15,50}$ which includes both internal friction for intact material and dry friction for damaged material. Motivated by the 
observed round shape of (10) Hygiea, we used the simpler frictionless model, as the friction clearly did not play a major role in the Hygiea-forming impact. For comparison, we also ran simulations with various friction coefficients.

During N-body simulations, we searched for particle collisions, performing either an inelastic bounce or merging of collided particles, depending on their relative velocities and the spin rate of the merger. When particles merged, the resulting volume, velocity and spin rate of the merger was determined to conserve the total volume, momentum and angular momentum. Overlapping particles were treated the same way as collided particles; as we performed a late hand-off when relative velocities of particles inside individual fragments were already small, the respective particles underwent a quick merging and a precise handling of overlaps was not needed. Although merging erased the shape information, here we are only interested in fragment sizes and merging is thus a viable option.

\section{Rheology in SPH simulations}

In the simulations presented in the main text, we use the von Mises criterion. The yield stress is computed using $Y=(1-D) Y_{0}$, were $\mathrm{Y}_{0}$ is a material-specific, but pressure-independent constant and $D$ is the scalar damage. In this model, fully damaged material experiences no friction and essentially behaves as a fluid.

To model friction of granular material (which would be especially important for asteroids and impacts much smaller than in Hygiea's case), we also implemented the Drucker-Prager rheology ${ }^{15,50}$ in our code. It defines the yield strength of intact material as:

$$
Y_{i}=Y_{0}+\frac{\mu_{i} P}{1+\mu_{i} P /\left(Y_{m}-Y_{0}\right)}
$$


where $\mu_{\mathrm{i}}$ is the coefficient of internal friction, $Y_{0}$ the cohesion (yield strength at zero pressure) and $Y_{\mathrm{m}}$ the von Mises plasticity limit. For fully damaged rock, the yield strength is proportional to the pressure:

$$
Y_{d}=\mu_{d} P
$$

where $\mu_{\mathrm{d}}$ is the coefficient of dry friction, which is related to the angle of repose. In the intermediate state where $0<D<1$, the yield strength is given by a linear interpolation, $Y=(1-D) Y_{i}+D Y_{d}$

The final shape of the largest remnant is affected by the coefficient of dry driction. However, using the model with non-negligible friction, $\mu_{\mathrm{d}}>0.1$, yields a very poor match to the observed round shape of (10) Hygiea (see Supplementary figure 10). This issue has been previously recognized by studies of cratering events ${ }^{24,25}$ and is commonly explained by introducing the acoustic fluidization. In the block model of acoustic fluidization, yield strength is further modified as:

$$
Y_{v i b}=\mu_{d}\left(P-P_{v i b}\right)+\eta_{l} \varrho \dot{\epsilon}
$$

where $P_{\text {vib }}$ is the vibrational pressure, calculated from the maximum vibrational particle velocity $^{51}, \eta_{1}$ the effective viscosity of fluidized material, $\dot{\epsilon}$ the strain rate. The vibrational velocity is exponentially attenuated after the impact, however, the time scale of this process is a free parameter. Instead of using the block model directly, we prefer the von Mises model, with a similar free parameter, i.e. the time scale of acoustic fluidization after which the body regains its strength. This model matches the observed shape very well (see main text Figure 3 and Supplementary Figure 10).

\section{Parameters of the SPH simulations}


We considered both the target and the impactor to be monolithic bodies with an initial density of the material $\rho_{0}=2000 \mathrm{~kg} / \mathrm{m}^{3}$, corresponding to the present-day density of Hygiea. We assumed material properties of basalt ${ }^{14,16}$. The pressure and the sound speed were determined using the Tillotson's equation of state, assuming bulk modulus $\mathrm{A}=2.67 \times 10^{10} \mathrm{~Pa}$, and specific energies for incipient and complete vaporization $\mathrm{u}_{\mathrm{iv}}=4.72 \times 10^{6} \mathrm{~J} / \mathrm{kg}$ and $\mathrm{u}_{\mathrm{cv}}=1.82 \times 10^{7} \mathrm{~J} / \mathrm{kg}$, respectively. The strength model used the von Mises yield criterion with shear modulus $\mu=$ $2.27 \times 10^{10} \mathrm{~Pa}$, elasticity limit $\mathrm{Y}_{0}=3.5 \times 10^{9} \mathrm{~Pa}$ and specific melting energy $\mathrm{u}_{\text {melt }}=3.4 \times 10^{6} \mathrm{~J} / \mathrm{kg}$. To account for material fragmentation, we used the Grady-Kipp model with Weibull coefficient $\mathrm{k}=4 \times 10^{29}$ and Weibull exponent $\mathrm{m}=9$. In our simulations, the target had $\mathrm{N} \sim$ $4 \times 10^{5}$ particles, the spatial resolution being therefore around $\sim 6 \mathrm{~km}$ which is sufficient to resolve hundreds of the family members. The number of particles for the impactor was chosen so as to obtain the same particle density as the target. The equations were integrated using a predictor-corrector method, time step of which has been limited by the CFL criterion with Courant number $\mathrm{C}=0.2$. A subset of our simulations and the used paraemters are diplayed in supplementary figure 5. Finally, the cumulative size-frequency distributions (SFD) of synthetic families are compared to the SFD of the observed Hygiea family in supplementary Figure 11.

\section{Data availability}

As soon as papers for our large program are accepted for publication, we make the corresponding reduced and deconvolved AO images and 3D shape models publicly available at http://observations.lam.fr/astero/.

\section{Code availability}


The code used to generate the 3D shape is freely available at https:/github.com/matvii/ADAM. The code used to perform the SPH simulations is freely available at https://gitlab.com/sevecekp/sph.

\section{References}

1. Takir, D. \& Emery, J. P. Outer Main Belt asteroids: Identification and distribution of four 3- $\mu \mathrm{m}$ spectral groups. Icarus 219, 641-654 (2012).

2. Vernazza, P., Castillo-Rogez, J., Beck, P., et al. Different Origins or Different Evolutions? Decoding the Spectral Diversity Among C-type Asteroids. Astron. J. 153, 10 pp. (2017).

3. Carruba, V., Domingos, R. C., Huaman, M. E., Santos, C. R. dos, Souami, D. Dynamical evolution and chronology of the Hygiea asteroid family. Mon. Not. R. Astron. Soc. 437, 2279$2290(2014)$.

4. Vernazza, P., Brož, M., Drouard, A., Hanuš, J., Viikinkoski, M., et al. The impact crater at the origin of the Julia family detected with VLT/SPHERE? A\&A 618, 16 pp. (2018).

5. Thalmann, C., Schmid, H. M., Boccaletti, A., Mouillet, D., Dohlen, K., et al. SPHERE ZIMPOL: Overview and performance simulation. Ground-based and Airborne Instrumentation for Astronomy II, Proc. SPIE 7014 (2008). 
6. Fusco, T., Mugnier, L. M., Conan, J.-M., Marchis, F., Chauvin, G., et al. Deconvolution of astronomical images obtained from ground-based telescopes with adaptive optics. Adaptive Optical System Technologies II. Edited by Wizinowich, Peter L.; Bonaccini, Domenico. Proc. SPIE 4839, 1065-1075 (2003).

7. Fetick, R. Jorda, L., Vernazza. P, et al. Closing the gap between Earth-based and interplanetary mission observations: Vesta seen by VLT/SPHERE. $A \& A$ 623, A6 (2019).

8. Viikinkoski, M., Kaasalainen, M., \& Durech, J. ADAM: a general method for using various data types in asteroid reconstruction. $A \& A \mathbf{5 7 6}$ (2015).

9. Michalowski, T., Velichko, F. P., Lindgren, M. et al. The spin vector of asteriod 10 Hygiea. A\&A Suppl. Series 91, 53-59 (1991).

10. Chandrasekhar, R. Ellipsoidal Figures of Equilibrium. Dover Publications, New York (1987).

11. Park, R. S., Vaughan, A. T., Konopliv, A. S., et al. High-resolution shape model of Ceres from stereophotoclinometry using Dawn Imaging Data. Icarus 319, 812-827 (2019).

12. Nesvorný, D., Brož, M., Carruba, V. Identification and Dynamical Properties of Asteroid Families. Asteroids IV, Patrick Michel, Francesca E. DeMeo, and William F. Bottke (eds.), University of Arizona Press, Tucson, 297-321 (2015). 
13. Thomas, P. C., Binzel, R. P., Gaffey, M. J., Storrs, A. D., Wells, E. N., Zellner, B. H. Impact excavation on asteroid 4 Vesta: Hubble Space Telescope results. Science 277, 14921495 (1997).

14. Benz, W. \& Asphaug, E. Impact simulations with fracture. I - Method and tests. Icarus 107, 98 (1994).

15. Jutzi, M., Holsapple, K., Wünneman, K., Michel, P. Modeling asteroid collisions and impact processes. Asteroids IV P. Michel, F. E. DeMeo and W. F. Bottke (eds), University of Arizona Press, Tucson, 679-699 (2015).

16. Ševeček, P.; Brož, M.; Nesvorný, D.; Enke, B.; Durda, D.; Walsh, K.; Richardson, D. C. SPH/N-Body simulations of small $(\mathrm{D}=10 \mathrm{~km})$ asteroidal breakups and improved parametric relations for Monte-Carlo collisional models. Icarus 296, 239-256 (2017).

17. Tillotson, J. H. Metallic equations of state for hypervelocity impact. Gen. Atomic Rep GA-3216 (1962).

18. von Mises, R. Mechanik der festen körper im plastisch- deformablen zustand. Nachrichten von der Gesellschaft der Wissenschaften zu Göttingen, Mathematisch-Physikalische Klasse 1913, 582-592 (1913). 
19. Grady, D. \& Kipp, M. Continuum modelling of explosive fracture in oil shale. Int. J. of Rock Mechanics and Mining Sciences \& Geomechanics Abstracts 17, 147 - 157 (1980).

20. Barnes, J. \& Hut, P. A hierarchical O(N $\log \mathrm{N})$ force-calculation algorithm. Nature 324, 446-449 (1986).

21. Michel, P., Benz, W., Tanga, P., Richardson, D. C. Collisions and Gravitational Reaccumulation: Forming Asteroid Families and Satellites. Science 294, 1696-1700 (2001).

22. Tanga, P., Hestroffer, D., Delbo, M., Richardson, D.C., Asteroid rotation and shapes from numerical simulations of gravitational re-accumulation. Planet. Space Sci. 57, 193-200 (2009).

23. Melosh, H. J. \& Ivanov, B. A. Impact crater collapse. AREPS 27, 385-415 (1999).

24. Riller U., Poelchau, M. H., Rae, A.S. P., Bednarczyk, T., Collinset, G. S., et al. Rock fluidization duringpeak-ring formation of large impact structures. Nature 561, 511-518 (2018).

25. Jutzi, M., Asphaug, E., Gillet, P., Barrat, J.-A., Benz, W. The structure of the asteroid 4 Vesta as revealed by models of planet-scale collisions. Nature 494, 207-210 (2013). 
26. Wadell, H. Volume, Shape and Roundness of Quartz Particles. Journal of Geology 43, 250-280 (1935).

27. Warner, B. D., Harris, A. W., Pravec, P. The asteroid lightcurve database. Icarus 202, 134-146 (2009).

28. Jehin, E., Gillon, M., Queloz, D., Magain, P., Manfroid, J., et al. TRAPPIST: TRAnsiting Planets and PlanetesImals Small Telescope. The Messenger 145, 2-6 (2011).

29. Pettengill, G. H., Ford, Peter G., Johnson, W. T. K., Raney, R. K., Soderblom, L. A. Magellan - Radar performance and data products, Science 252, 260-265 (1991).

30. Thomas, P. C., Veverka, J., Simonelli, D., Helfenstein, P., Carcich, B., et al. The shape of Gaspra, Icarus 107, 23 (1994).

31. Hudson, R. S., Ostro, S. J., Jurgens, R. F., Rosema, K. D., Giorgini, J. D., et al. Asteroid radar shape models, 6489 Golevka, NASA Planetary Data System, id. EAR-A-5-DDRRADARSHAPE-MODELS-V1.1:RSHAPES-6489GOLEVKA-200006 (2000).

32. Ostro, S. J., Rosema, K. D., Hudson, R. S., Jurgens, R. F., Giorgini, J. D., et al. Asteroid radar shape models, 1620 Geographos, NASA Planetary Data System, id. EAR-A-5-DDRRADARSHAPE-MODELS-V1.1:RSHAPES-1620GEOGRAPHOS-200006 (2000). 
33. Smith, D.E., Zuber, M.T., Frey, H.V., Garvin, J.B., Head, J.W., et al. Mars Orbiter Laser Altimeter-Experiment summary after the first year of global mapping of Mars, J. Geophys. Res. 106(E10), 23,689-23, 722 (2001).

34. Jorda, L., Lamy, P. L., Gaskell, R. W., Kaasalainen, M., Groussin, O., Besse, S., Faury, G. Asteroid (2867) Steins: Shape, topography and global physical properties from OSIRIS observations, Icarus 221, 1089-1100 (2012).

35. Preusker, F., Scholten, F., Matz, K., Roatsch, T., Jaumann, R., et al. Stereophotogrammetrically derived topography of asteroid (4) Vesta, American Geophysical Union, Fall Meeting 2012, abstract id.P43E-05 (2012).

36. Jaumann, R., Williams, D. A., Buczkowski, D. L., Yingst, R. A., Preusker, F., et al. Vesta's Shape and Morphology, Science 336, 687-690 (2012).

37. Farnham, T.L. Shape model of asteroid 21 Lutetia, RO-A-OSINAC/OSIWAC-5LUTETIA-SHAPE-V1.0, NASA Planetary Data System (2013).

38. Preusker, F., Stark, A., Oberst, J., Becker, K. J., Perry, M. E., Solomon, S. C. Topography of Mercury: A global model from MESSENGER orbital stereo mapping, European Planetary Science Congress (2014). 
39. Preusker, F., Scholten, F., Matz, K.-D., Elgner, S., Jaumann, R., et al. Dawn at Ceres Shape Model and Rotational State, 47th Lunar and Planetary Science Conference, held March 21-25, 2016 at The Woodlands, Texas. LPI Contribution No. 1903, p. 1954 (2016).

40. Viikinkoski, M., Vernazza, P., Hanuš, J., Le Coroller, H., Tazhenova, K., et al. (16) Psyche: A mesosiderite-like asteroid? $A \& A$ 619, L3 (2018).

41. Hanuš, J., Marsset, M., Vernazza, P., Viikinkoski, M., Drouard, A., et al. The shape of (7) Iris as evidence of an ancient large impact? $A \& A \mathbf{6 2 4}$ (2019).

42. Hiesinger, H., Marchi, S., Schmedemann, N., Schenk, P., Pasckert, J. H., et al. Cratering on Ceres: Implications for its crust and evolution. Science 353 (2016).

43. Bland, M. T., Raymond, C. A., Schenk, P. M., Fu, R. R., Kneissl, T. et al. Composition and structure of the shallow subsurface of Ceres revealed by crater morphology. Nat. Geosci. 9, 538-542 (2016).

44. Knezevic, Z. \& Milani, A. Proper element catalogs and asteroid families. $A \& A$ 403, $1165-1173$ (2003).

45. Zappala, V., Cellino, A., Farinella, P. \& Milani, A. Asteroid families. 2: Extension to unnum- bered multiopposition asteroids. Astron. J. 107, 772-801 (1994). 
46. Ivezic, $Z^{\longleftarrow}$. et al. Solar System Objects Observed in the Sloan Digital Sky Survey Commissioning Data. Astron. J. 122, 2749-2784 (2001).

47. Nugent, C. R. et al. NEOWISE Reactivation Mission Year One: Preliminary Asteroid Diam-eters and Albedos. Astrophys. J. 814, 117 (2015).

48. Usui, F. et al. Asteroid Catalog Using Akari: AKARI/IRC Mid-Infrared Asteroid Survey. Pub. Astron. Soc. Japan 63, 1117-1138 (2011).

49. Schäfer, C., Riecker, S., Maindl, T. I., Speith, R., Scherrer, S., et al. A smooth particle hydrodynamics code to model collisions between solid, self-gravitating objects. $A \& A \mathbf{5 9 0}$, A19 (2016).

50. Collins, G. S. and Melosh, H. J. and Ivanov, B. A. Modeling damage and deformation in impact simulations. Met. Planet. Sci. 39 (2004).

51. Silber, E.A., Osinski, G.R., Johnson, B.C., Grieve, R.A.F. Effect of impact velocity and acoustic fluidization on the simple-to-complex transition of lunar craters. J. Geophys. Res. (Planets) 122, 800 (2017).

52. Carry, B. Density of Asteroids. Planet. Space Sci. 73, 98 (2012). 
53. Vasiliev, M. V. and Yagudina, E. I. Determination of masses for 26 selected minor planets from analysis of observations their mutual encounters with asteroids of lesser mass. Communications of IAA of RAS (1999).

54. Krasinsky, G. A., Pitjeva, E. V., Vasiliev, M. V., Yagudina, E. I. Estimating masses of asteroids. Communications of IAA of RAS (2001).

55. Michalak, G. Determination of asteroid masses. II. (6) Hebe, (10) Hygiea, (15) Eunomia, (52) Europa, (88) Thisbe, (444) Typtis, (511) Davida and (704) Interamnia. $A \& A$ 374, 703711, (2001).

56. Chernetenko, Y. A. and Kochetova, O. M. Masses of some large minor planets. Asteroids, Comets, and Meteors, 437-440 (2002).

57. Kochetova, O. M. Determination of Large Asteroid Masses by the Dynamical Method. Solar System Research 38, 66-75 (2004).

58. Chesley, S. R., Owen, Jr., W. M., Hayne, E. W., Sullivan, A. M., Dumas, R. C., et al. The Mass of Asteroid 10 Hygiea. Bulletin of the American Astronomical Society 524 (2005).

59. Aslan, Z., Gumerov, R., Hudkova, L., Ivantsov, A., Khamitov, I., et al. Mass Determination of Small Solar System Bodies with Ground-based Observations. Astronomical Society of the Pacific Conference Series 370, 52-58 (2007). 
60. Baer, J. and Chesley, S. R. Astrometric masses of 21 asteroids, and an integrated asteroid ephemeris. CMDA 100, 27-42 (2008).

61. Ivantsov, A. Asteroid mass determination at Nikolaev Observatory. Planet. Space Sci. 56, 1857-1861 (2008).

62. Folkner, W. M. and Williams, J. G. and Boggs, D. H. The Planetary and Lunar Ephemeris DE 421. IPN Progress Report 42, 1-34 (2009).

63. Pitjeva, E. V. EPM ephemerides and relativity. IAU Symposium 26, 170-178 (2010).

64. Baer, J. and Chesley, S. R. and Matson, R. D. Astrometric Masses of 26 Asteroids and Observations on Asteroid Porosity. Astron. J. 141, 143-155 (2011).

65. Konopliv, A. S., Asmar, S. W., Folkner, W. M., Karatekin, O., Nunes, D. C., et al. Mars high resolution gravity fields from MRO, Mars seasonal gravity, and other dynamical parameters. Icarus 211, 401-428 (2011).

66. Zielenbach, W. Mass Determination Studies of 104 Large Asteroids. Astron. J. 142, 120$128(2011)$. 
67. Fienga, A., Kuchynka, P., Laskar, J., Manche, H. and Gastineau, M. Asteroid mass determinations with INPOP planetary ephemerides. EPSC-DPS Joint Meeting (2011).

68. Fienga, A., Manche, H., Laskar, J., Gastineau, M. and Verma, A. INPOP (2012).

69. Kuchynka, P. and Folkner, W. M. A new approach to determining asteroid masses from planetary range measurements. Icarus 222, 243-253 (2013).

70. Fienga, A., Manche, H., Laskar, J., Gastineau, M. and Verma, A. INPOP (2014).

71. Goffin, E. Astrometric asteroid masses: a simultaneous determination. $A \& A \mathbf{5 6 5}$ (2014).

72. Kochetova, O. M. and Chernetenko, Y. A. Mass determinations for 27 asteroids by the dynamic method. Solar System Research 48, 295-301 (2014).

73. Viswanathan, V. and Fienga, A. and Gastineau, M. and Laskar, J. INPOP17a planetary ephemerides. Notes Scientifiques et Techniques de l'Institut de mécanique celeste 108 (2017).

74. Siltala, L. and Granvik, M. Asteroid mass estimation using Markov-chain Monte Carlo. Icarus 297, 149-159 (2017). 
75. Baer, J. and Chesley, S. R. Simultaneous Mass Determination for Gravitationally Coupled Asteroids. Astron. J. 154, 76 (2017).

76. Fienga, A., et al.. INPOP (2018).

\section{Acknowledgments:}

P.V., A.D., and B.C. were supported by CNRS/INSU/PNP. M.B. was supported by the grant 18-04514J of the Czech Science Foundation. J.H. and J.D. were supported by the grant 1809470S of the Czech Science Foundation and by the Charles University Research Programme No. UNCE/SCI/023. This project has received funding from the European Union's Horizon 2020 research and innovation programmes under grant agreement No. 730890 and 687378 . This material reflects only the authors' views and the Commission is not liable for any use that may be made of the information contained herein. TRAPPIST-North is a project funded by the University of Liège, in collaboration with Cadi Ayyad University of Marrakech (Morocco). TRAPPIST-South is a project funded by the Belgian Fonds (National) de la Recherche Scientifique (F.R.S.-FNRS) under grant FRFC 2.5.594.09.F. E.J and M.G are F.R.S.-FNRS Senior Research Associates.

\section{Author contributions}

P.V. designed the research . P.V., M.M, R.F. and T.F. reduced and deconvolved the SPHERE images. M.V. and J.H. reconstructed the 3D shape of Hygiea. L.J and P.V. performed the analysis of Hygiea's shape. P.S. and M.B. ran the SPH simulations. M.F. and E.J. acquired and reduced the TRAPPIST data. M.M and L.J. produced the albedo map. P.V. and F.D. served as principal investigators to acquire the near-infrared spectral data. B.C. provided the 
mass estimate. P.V., L.J., P.S. and M.B. worked jointly to write the manuscript. All authors discussed the results and commented on the manuscript.

\section{Competing interests}

The authors declare no competing interests

\section{Additional information}

Correspondence and requests for materials should be addressed to P.V. 


\section{Figures}

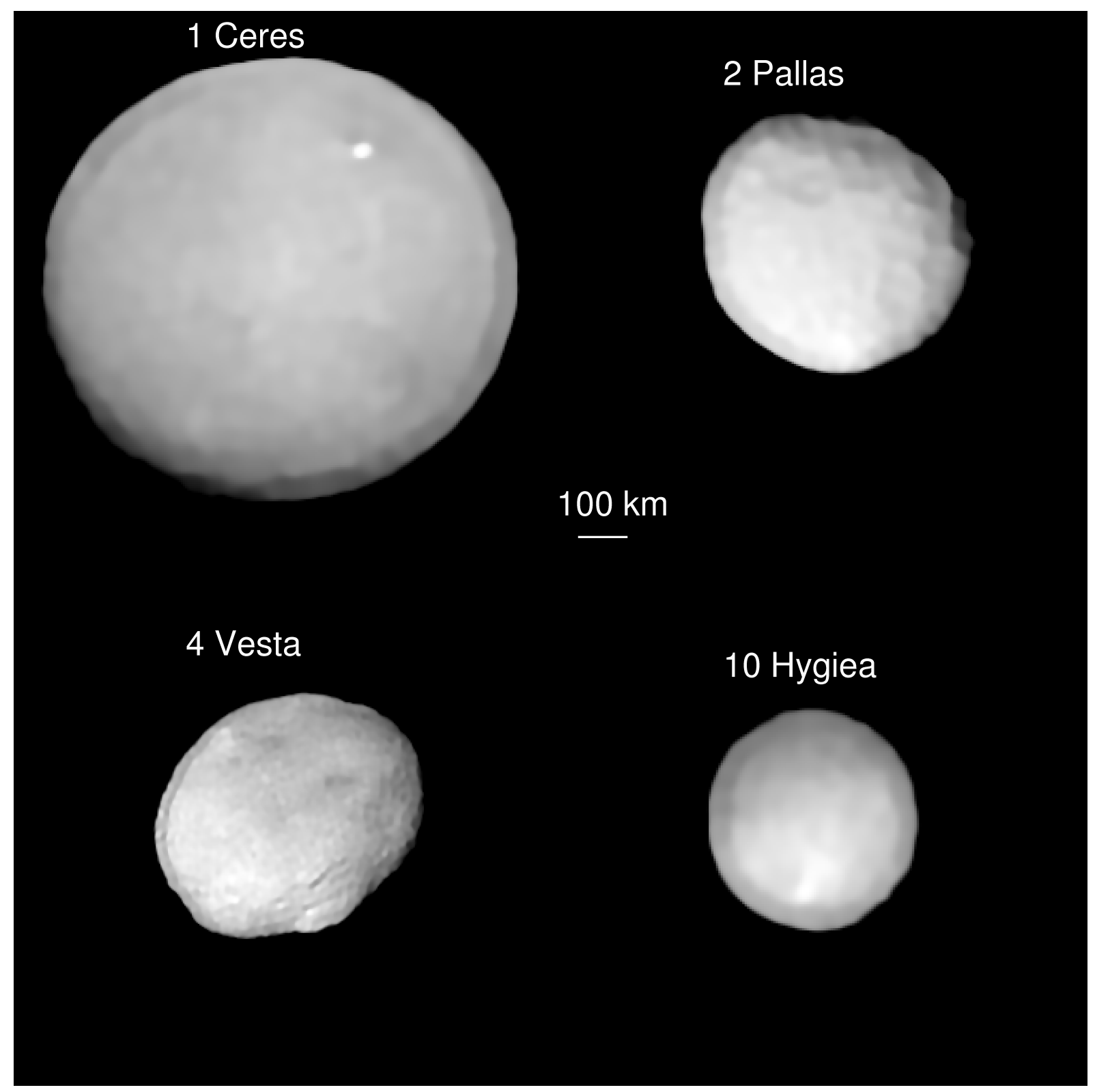

Figure 1: VLT/SPHERE deconvolved images of the four largest main belt objects. The relative sizes are respected and the scale is indicated on the plot. 


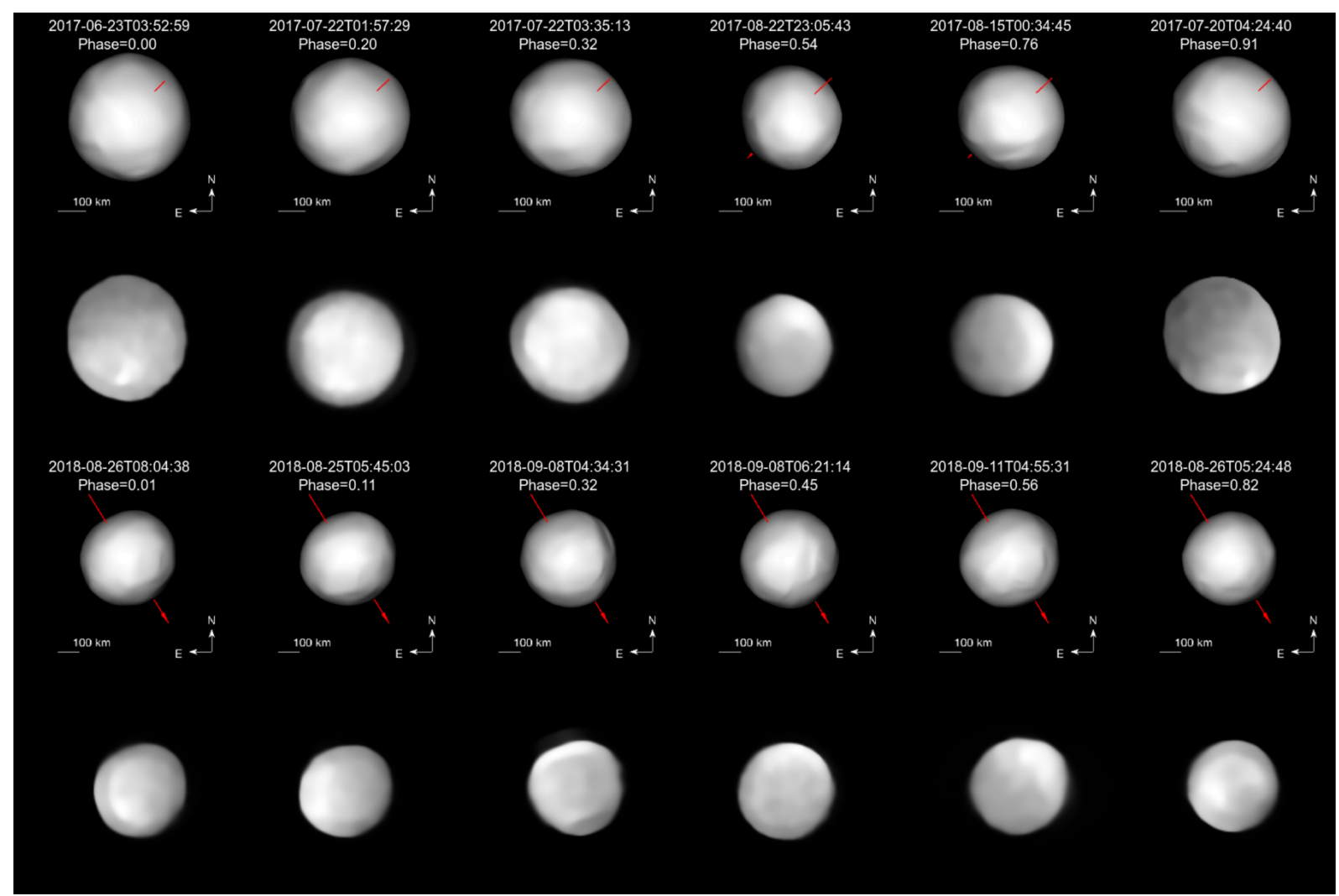

Figure 2: Comparison between the deconvolved images of Hygiea (bottom panels) and the corresponding shape model projections (top panels). Hygiea's spin axis (red) is also shown. 


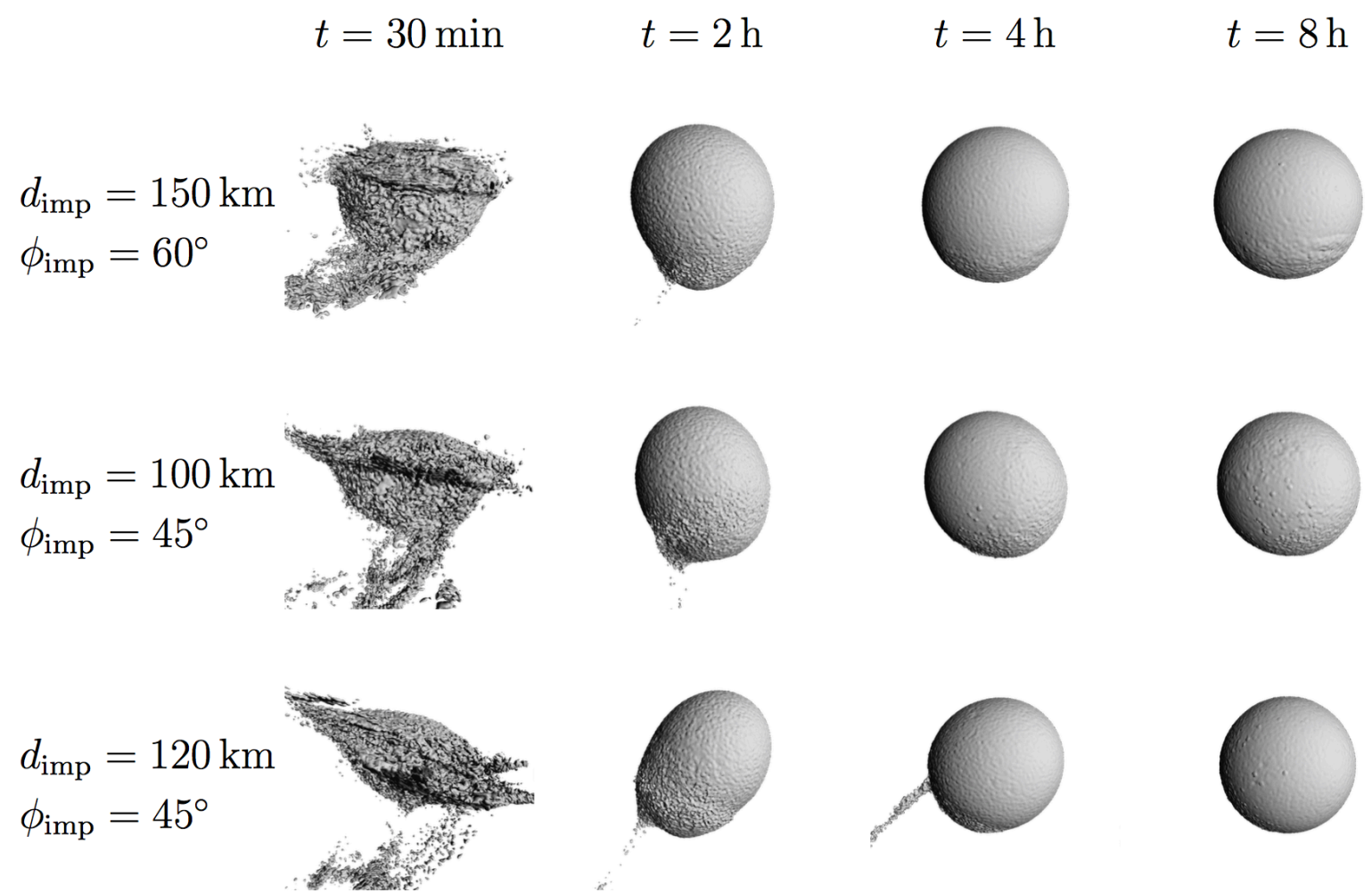

Figure 3: SPH simulations reveal a nearly spherical shape for Hygiea following postimpact reaccumulation. SPH simulations were ran to simulate the giant collision at the origin of the prominent Hygiea family with a focus on the post-impact shape of the largest remnant, namely Hygiea. For an accurate representation of the surface, we generated it as an isosurface of the density using the ray marching algorithm, rather than rendering individual $\mathrm{SPH}$ particles. At time $\mathrm{t}=30 \mathrm{~min}$, Hygiea is fully fragmented and significantly deformed. Shortly after, most of the ejected material reaccumulates on Hygiea. Finally, macroscopic oscillations are suppressed and Hygiea reaches a nearly spherical equilibrium shape. No large crater has been preserved. 


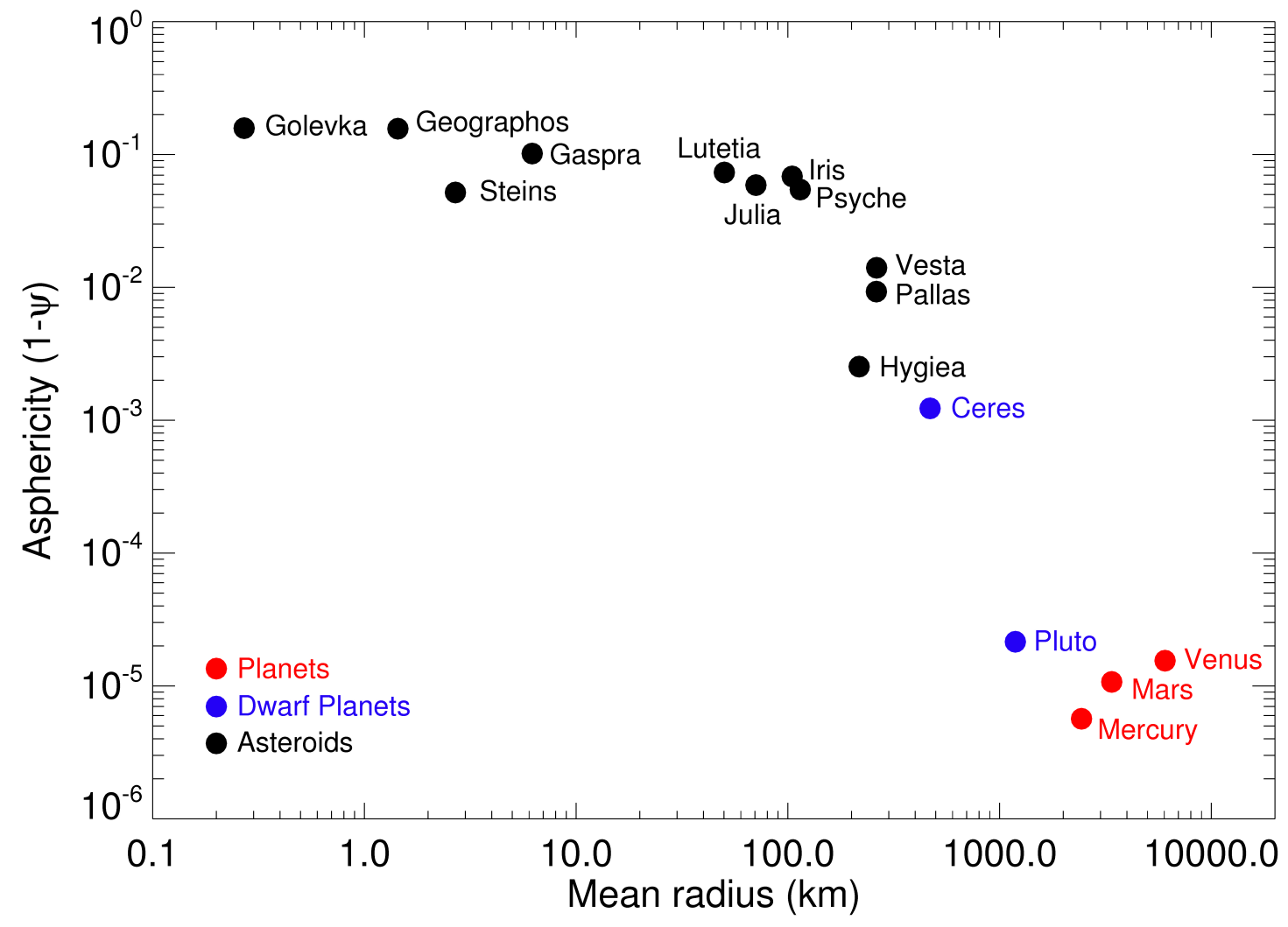

Figure 4: Asphericity of solar system objects as a function of their mean radius. The parameter $\psi$ corresponds to the sphericity index (Wadell 1935) applied to spherical harmonics developments of the 3D shape models of each object. Hygiea appears nearly as spherical as dwarf planet Ceres. 